to the tracheal bifurcation following a natural anatomical angle. Thus the importance of not pushing the tube down too far, which can be avoided by choosing a correct length of tube, can be demonstrated.

Two detachable "lungs" provide demonstration facilities for mouth-to-tube resuscitation, selfinflating bag resuscitation, endotracheal suctioning, or visualizing intubation of the right bronchus. A warning buzzer will sound if the laryngoscope is levered against the upper teeth. Pressure on to the
larnynx can be simulated, permitting the demonlarnynx can be simulated, permitting the demon-
stration of this useful manoeuvre often needed to stration of this useful manoeuvre often needed to the larynx is lying in anterior position.

A unique feature of the intubation trainer is that it allows the simulation of varying degrees of difficulty for the trainee. Anterior or posterior positional movement of the larynx assembly in relation to the spine is adjustable, thereby permitting repeatable settings of degrees of difficulty mitting repeatable settings of degrees of difficulty from "very easy" to "difficult." Supplementing the apparatus is a set of teaching cards which supply,
with teaching notes, the necessary information to perform the manoeuvre in the correct manner.

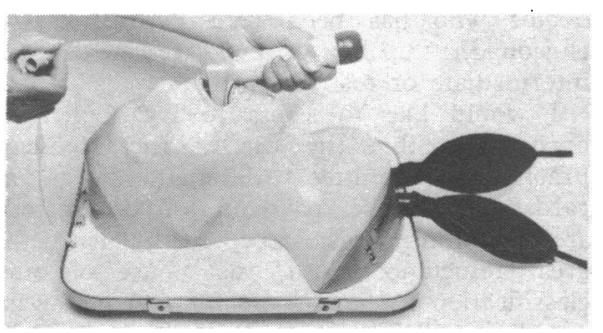

Though primarily designed for teaching by experts to small classes, the nature of the presentation is readily amenable to selfteaching, the student following the description booklet and the teaching cards and practising on the intubation trainer until he understands the manipulation and achieves repeated intubation success.

While it cannot be claimed that the ability to intubate the "trachea" of a stimulator satisfactorily means proficiency in the human situation, nevertheless the dexterity required and the understanding of the principles involved for intubation permit a highly informed approach to the "live" event. Medical students who had been trained by the teaching scheme using the Laerdal model (the only alternative commercial apparatus) displayed a ready expertise when faced with endotracheal intubation on the anaesthetized patient. On the normal adult patient most students were able to perform intubation satisfactorily and without tissue damage or undue delay on their first attempt.

The scheme, known as the Royal Free Hospital Endotracheal Intubation Training Scheme, complete with intubation simulator, instruction manual and display teaching cards, is being commercially prepared and distributed by Vitalograph Limited of Buckingham.

-I am, etc.,

Royal Free Hospital,

T. Hillary Howells

London N.W.3

Howells, T. H., Emery, F. M., and Twentyman, J5, E. C., British foumal of Anaesthesia, 1973

Psychiatric Referral in the General Hospital

SIR,-Dr. G. P. Maguire and his colleagues (16 February, p. 268) have described an interesting investigation of psychiatric morbidity and referral in the medical ward. However, in discussing factors affecting referral there is some confusion between those likely to be related to re cognition of psychiatric illness and others which may then influence the decision to refer. A record of past psychiatric illness would seem to fall into the first category while severity of psychiatric disturbance is probably related not only to recognition of illness but also to the choice of which ill patients to refer. The only evidence specifically related to the second category concerns the $26 \%$ referral of patients whose notes indicated an awareness of psychiatric disturbance. Referral was "very strongly determined by how fas their behaviour had obtruded or created problems for the medical staff"-a disappointing reflexion on the relative emphasis of management problems over clinical factors in the decision to refer.

A more optimistic picture, however, was revealed by study ${ }^{1}$ by $M$. J. Pritchard of factors related to psychiatric referral in this general hospital. Here the comparison was between referned and non-referred patients, all of whom were diagnosed (that is, recognized) as psychiatrically ill. Referral occurred in about a third of the 252 cases and was found to be related to such factors as age, type of psychiatric illness, and the extent to which it was due to organic disease, as well as the existence of a suicidal attempt. Previous psychiatric contact within this group was inversely related to referral-a possible interpretation being that information from the earlier psychiatric assessment was often considered to be sufficient for the present admission.

From these results it appeared that referral was not a matter of chance but was being influenced by identifiable and valid clinical factors. At the same time, however, considerable differences were found between physicians in their use of psychiatric referral. This correlated with other variables thought to reflect the psychiatric awareness of the physician and/or his relationship with the psychiatric department, pointing again to the value of liaison work for the psychiatrist in the general hospital.-I am, etc.

Department of Psychiatry,

M. J. PrItchard

Tondon E.1 Pritchard, M. J., Postgraduate Medical fournal,
1972, 48, 645.

\section{Co-trimoxazole Resistance}

SIR,-In the recent correspondence about co-trimoxazole resistance Dr. R. W. Lacey and Mrs. Evelyn L. Lewis (20 October, p. 164) referred to our report of thyminerequiring mutants isolated from infected unine and Drs. R. Then and P. Angehrn (12 January, p. 78) quoted our findings in support of their view that "the selection of thy mutants in vivo which are able to survive and are due to the use of co-trimoxazole is obviously a rare event of little importance for clinical therapy." Perhaps our experience may help to clarify some of the oints raised in these letters.

The three strains of thy mutants of Proteus mirabilis which we isolated from infected urine in 1971 and 1972 alented us to the existence of the phenomenon, but despite a very careful watch since 1972 only one Echerichia coli thy- mutant has been isolated from over 75,000 urines examined. The patient had renal calculi with gross pyuria, as did the other three pacients pre- viously reported,' and had been given cotrimoxazole for long-term suppressive therapy. The thy- mutants isolated from these patients were all stable in vituro and have not reverted to normal wild type organisms in up to three years of preservation by subcultures on Dorsett egg medium. In all our patients the thy- mutants disappeared from the urine and were replaced by normal organisms after cessation of cotrimoxazole therapy. These findings suggest to us that the thy mutants are rare and can be associated with certain clinical situations, particularly infected renal calculi treated with long-term co-trimoxazole.

It is possible that the urine of these patients may have contained a quantity of thymine sufficient to maintain the growth of the mutants, and this could be related to the pyuria. However, we have treated over 100 patients with infection and pyuria, but no stones, with long-term co-trimoxazole in similar dosage and have not encountered thy mutants. It is well recognized that live organisms persist in the matrix of infected calculi, ${ }^{2}$ and therefore they could be exposed to co-trimoxazole for very much longer than is the case in infection without stones. A simiiar situation could obtain in other clinical conditions, and it is interesting that Dr. Lacey and Mrs. Lewis have recently isolated a thymidine-requiring strain of Staphylococcus aureus from the sputum of a child with fibrocystic lung disease who had been treated with cotrimoxazole. Live organisms could have persisted in the scar tissue allowing the mutation to occur.

We have also observed in vitro that $E$. coli NCTC 10418 and other strains of $E$. coli and $P$. mirabilis can produce a growth factor which enables these organisms to grow on minimal agar without thymine. If this were the case in vivo, one would not need to postulate pus cells as the sounce of thymine or thymidine. Persistence of live organisms in the stone might suffice.

Finally we think that the emengence of thy mutants in the urine of these patients represents failure to co-trimoxazole therapy, and it is impontant therefone to be aware of their existence.-We are, etc.

\section{O. A. Orubadejo}

R. M. MASKELI

Public Health Laboratory,

St. Mary's General Hospital,
Portsmouth

1 Okubadjo, O. A., and Maskell, R. M., Fournal of General Microbiology, 1973, 77, 533.

2 Nemoy, N. J., and Stamey, T. A.' Fournal of the
American Medical Association, 1971, 215, 1470 .

\section{The Solitary Thyroid Nodule}

SIR,-Your leading article on this subject (10 November, p. 310) was the second in as many years (25 September 1971, p. 720) and indicates continuing interest in this problem. The more recent article rightly points out the different incidence of malignancy in vanious parts of the world. However, neither mentions the signifioance of sex in relation to the incidence of malignancy. There appears to be such a relationship in South India, where the overall incidence of malignancy in solitary nodules is comparatively high.

In our institution analysis 1 of 178 thyroid nodules considered to be "solitary" at operation disclosed 62 carcinomas (25 in 